\title{
ON THE PROBLEM OF LINEARIZATION FOR STATE-DEPENDENT DELAY DIFFERENTIAL EQUATIONS
}

\author{
KENNETH L. COOKE AND WENZHANG HUANG \\ (Communicated by Hal L. Smith)
}

\begin{abstract}
The local stability of the equilibrium for a general class of statedependent delay equations of the form

$$
\dot{x}(t)=f\left(x_{t}, \int_{-r_{0}}^{0} d \eta(s) g\left(x_{t}\left(-\tau\left(x_{t}\right)+s\right)\right)\right)
$$

has been studied under natural and minimal hypotheses. In particular, it has been shown that generically the behavior of the state-dependent delay $\tau$ (except the value of $\tau$ ) near an equilibrium has no effect on the stability, and that the local linearization method can be applied by treating the delay $\tau$ as a constant value at the equilibrium.
\end{abstract}

\section{INTRODUCTION}

Differential delay equations or functional differential equations have been used in the modeling of scientific phenomena for many years. Often it has been assumed that the delays are either fixed constants or are given as integrals (distributed delays). However, in recent years, the more complicated situation in which the delays depend on the unknown functions has been proposed in models $[1,2,3,4$, $5,6,7,9,10,11,12,13]$. These equations are frequently called equations with state-dependent delay. For example, it is a well-known fact that many types of structured population models can be reduced to the study of functional differential equations and in some cases this reduction yields a delay equation of threshold type. For examples of these models and equations, see the references below.

Although state-dependent equations often are functional differential equations of standard type, there are instances in which they are not, see [13], or in which it would be useful to have results specific to this situation. The purpose of this note is to show how to obtain a formal linearization around an equilibrium of a quite general type of equation with state-dependent delay, and to give a proof of this result under natural and minimal hypotheses. As will be shown, the result is easy to understand and use. The result then provides the means to study the

Received by the editors December 3, 1993.

1991 Mathematics Subject Classification. Primary 34K20.

The first author's research was supported in part by NSF grant DMS 9208818.

The second author's research was supported in part by NSF grant DEB 925370 to Carlos Castillo-Chavez and by the U.S. Army Research Office through the Mathematical Science Institute of Cornell University. 
asymptotic stability of an equilibrium of a state-dependent functional differential equation, and the question of Hopf bifurcation from the equilibrium.

\section{Formal LINEARIZATION AND STABILITY}

In this paper we consider the following type of state-dependent FDE:

$$
\dot{x}(t)=F\left(x_{t}\right), \quad x_{0}=\phi \in C=C\left([-r, 0], \mathbb{R}^{n}\right),
$$

where $x_{t} \in C$ is defined by $x_{t}(s)=x(t+s), s \in[-r, 0], F$ takes the form

$$
F(\phi)=f\left(\phi, \int_{-r_{0}}^{0} d \eta(s) g(\phi(-\tau(\phi)+s))\right),
$$

$f: C \times \mathbb{R}^{n} \rightarrow \mathbb{R}^{n}, g: \mathbb{R}^{n} \rightarrow \mathbb{R}^{n}$ are continuously differentiable and $\tau: C \rightarrow\left[0, r_{1}\right]$ $\left(r_{0}+r_{1} \leq r\right)$ is continuous, and $\eta$ is of bounded variation on $\left[-r_{0}, 0\right]$. The idea in taking $F(\phi)$ of this form was suggested by the form in Alt [2] and the examples in Section 4 .

By the continuity of $f, g$ and $\tau$, one is able to see that $F: C \rightarrow \mathbb{R}^{n}$ is continuous. Hence following a standard approach we conclude the local existence of a solution to Eq. (2.1). Nevertheless, if $\tau$ is not Lipschitzian, then $F(\phi)$ generally is not Lipschitz continuous and hence the solution to (2.1) may not be unique. The purpose of this paper is not to address the uniqueness of solution, but the local stability of an equilibrium. As we can see later on in our analysis, nonuniqueness of solution does not affect the local stability. The main reason is that a solution of (2.1) with $\phi$ being an equilibrium is unique.

Without loss of generality we suppose

$$
F(0)=f\left(0, \int_{-r_{0}}^{0} d \eta(s) g(0)\right)=0,
$$

that is, zero is a solution of (2.1). We note that $F$ will not be differentiable at $\phi=0$ in general if $\tau$ is not differentiable at $\phi=0$. To study the local stability of the zero solution we formally linearize (2.1) at zero by treating $\tau=\tau(0)$ as a constant to obtain a usual state-independent linear delay equation

$$
\dot{x}(t)=D_{\phi} f(0, \hat{\eta} g(0)) x_{t}+D_{y} f(0, \hat{\eta} g(0)) \int_{-r_{0}}^{0} d \eta(s) D g(0) x_{t}(-\tau(0)+s),
$$

where $\hat{\eta}=\int_{-r_{0}}^{0} d \eta(s)$.

The purpose of this paper is to establish the following result which shows that the stability of (2.2) does reflect the stability of the zero solution of the nonlinear equation (2.1). That is, Eq. (2.2) behaves like a linearization of (2.1) at zero.

Theorem 2.1. Let the linear operator $L: C \rightarrow \mathbb{R}^{n}$ be defined by

$$
L \phi=D_{\phi} f(0, \hat{\eta} g(0)) \phi+D_{y} f(0, \hat{\eta} g(0)) \int_{-r_{0}}^{0} d \eta(s) D g(0) \phi(-\tau(0)+s) .
$$

(i) If $\sup \left\{\operatorname{Re} \lambda\right.$ : $\left.\operatorname{det}\left(\lambda I-L e^{\lambda \cdot}\right)=0\right\}=-\alpha<0$, then for each small $\varepsilon>0$, there exist a constant $K(\varepsilon)$ and a neighborhood $V(\varepsilon) \subset C$ of the origin such that for $\phi \in V(\varepsilon)$, the solution $x_{t}(\phi)$ of $(2.1)$ is defined for $t \geq 0$ and

$$
\left\|x_{t}(\phi)\right\| \leq K(\varepsilon) e^{-(\alpha-\varepsilon) t}\|\phi\| .
$$


(ii) If $\sup \left\{\operatorname{Re} \lambda: \operatorname{det}\left(\lambda I-L e^{\lambda \cdot}\right)=0\right\}=\alpha>0$, then the zero solution is unstable. $^{1}$

If the equilibrium solution is $x^{*} \in \mathbb{R}^{n}$ instead of zero, so that

$$
F\left(x^{*}\right)=f\left(x^{*}, \int_{-r_{0}}^{0} d \eta(s) g\left(x^{*}\right)\right)=0,
$$

then the correct equation to replace $(2.2)$ is

$$
\dot{x}(t)=D_{\phi} f\left(x^{*}, \hat{\eta} g\left(x^{*}\right)\right) x_{t}+D_{y} f\left(x^{*}, \hat{\eta} g\left(x^{*}\right)\right) \int_{-r_{0}}^{0} d \eta(s) D g\left(x^{*}\right) x_{t}\left(-\tau\left(x^{*}\right)+s\right) .
$$

\section{Proof of Theorem 2.1}

Before proceeding to the proof of Theorem 2.1 we first introduce our notation and give some preliminaries which are needed later on.

1. For notational simplicity, the Euclidean norm in $\mathbb{R}^{n}$, supremum norm in $C$ and total variation of a function on $\left[-r_{0}, 0\right]$ are all denoted by "\| $\|$ ".

2. For $h>0, B_{h}:=\{\phi \in C ;\|\phi\| \leq h\}, B_{h}^{n}:=\left\{y \in \mathbb{R}^{n} ;\|y\| \leq h\right\}$.

3. A solution of $(2.1)$ is denoted by $x(t, \phi)$ or $x_{t}(\phi)$ if it is considered in phase space $\mathbb{R}^{n}$ or $C$, respectively.

Lemma 3.1. There exist $\delta>0$ and $M>0$ such that

$$
\left\|f\left(\phi, \int_{-r_{0}}^{0} d \eta(s) g(\phi(-\tau(\phi)+s))\right)\right\| \leq M\|\phi\|, \quad \phi \in B_{\delta} .
$$

Proof. Since $f(\phi, y)$ and $g(z)$ are continuously differentiable in neighborhoods of $(0, \hat{\eta} g(0))$ in $C \times \mathbb{R}^{n}$ and of 0 in $\mathbb{R}^{n}$, respectively, there are $\delta_{1}>0$ and $M_{1}>0$ such that

$$
\begin{aligned}
&\|f(\phi, y)\|=\|f(\phi, y)-f(0, \hat{\eta} g(0))\| \\
& \leq M_{1}(\|\phi\|+\|y-\hat{\eta} g(0)\|), \quad \phi \in B_{\delta_{1}}, y-\hat{\eta} g(0) \in B_{\delta_{1}}^{n}, \\
&\|g(z)-g(0)\| \leq M_{1}\|z\|, \quad z \in B_{\delta_{1}}^{n} .
\end{aligned}
$$

Let

$$
\delta=\min \left\{\delta_{1}, \frac{\delta_{1}}{M_{1}\|\eta\|}\right\} \quad \text { and } \quad M=M_{1}(1+\|\eta\|) .
$$

Then for $\phi \in B_{\delta}$, we have

$$
\begin{aligned}
\left\|\int_{-r_{0}}^{0} d \eta(s) g(\phi(-\tau(\phi)+s))-\hat{\eta} g(0)\right\| & =\left\|\int_{-r_{0}}^{0} d \eta(s)[g(\phi(-\tau(\phi)+s))-g(0)]\right\| \\
& \leq\|\eta\| M_{1}\|\phi\| \leq \delta<\delta_{1} .
\end{aligned}
$$

\footnotetext{
${ }^{1}$ We say that the zero solution is unstable if there is a $\delta>0$, such that for each $\varepsilon>0$, there is a $\phi \in C,\|\phi\| \leq \varepsilon$ and $t_{\phi}>0$ such that $x_{t}(\phi)$ exists for $0 \leq t \leq t_{\phi}$ and $\left\|x_{t_{\phi}}(\phi)\right\| \geq \delta$.
} 
Therefore

$$
\begin{aligned}
& \left\|f\left(\phi, \int_{-r_{0}}^{0} d \eta(s) g(\phi(-\tau(\phi)+s))\right)\right\| \\
& \quad \leq M_{1}\left(\|\phi\|+\left\|\int_{-r_{0}}^{0} d \eta(s)[g(\phi(-\tau(\phi)+s))-g(0)]\right\|\right) \\
& \quad \leq\left(M_{1}+\|\eta\| M_{1}\right)\|\phi\| \\
& \quad=M\|\phi\| .
\end{aligned}
$$

An immediate corollary of Lemma 3.1 is

Corollary 3.2. Let $0<\delta_{0} \leq \delta$ and $0<\delta^{*}<e^{-M r} \delta_{0}$ be any fixed numbers. If $\phi \in B_{\delta^{*}}$, then $x(t, \phi)$ exists for $t \in\left[-r, t_{\phi}\right)$ with $t_{\phi}>r$. Furthermore, $\left\|x_{t}(\phi)\right\| \leq$ $e^{M t}\|\phi\|<\delta_{0}$, for $t \in[0, r]$.

Proof. If the lemma is false for some $x(t, \phi)$, then by the continuity of $x(t)=x(t, \phi)$ and the fact that $\left\|x_{0}\right\|=\|\phi\| \leq \delta^{*}<\delta_{0}$, there must be a $t^{*} \in(0, r]$ such that

$$
\|x(t)\|<\delta_{0}, \quad t \in\left[-r, t^{*}\right), \quad \text { and } \quad\left\|x\left(t^{*}\right)\right\|=\delta_{0} .
$$

Let $\hat{x}(t)=\max _{-r \leq s \leq t}\{\|x(s)\|\},-r \leq t \leq t^{*}$; then we have

$$
0 \leq \hat{x}(t)<\delta_{0}, \quad t \in\left[-r, t^{*}\right), \quad \hat{x}\left(t^{*}\right)=\delta_{0} .
$$

On the other hand, since $x(t)$ satisfies the integral equation

$$
x(t)=\phi(0)+\int_{0}^{t} f\left(x_{s}, \int_{-r_{0}}^{0} d \eta(s) g\left(x_{s}\left(-\tau\left(x_{s}\right)+s\right)\right)\right) d s,
$$

by applying Lemma 3.1 we have

$$
\begin{aligned}
\|x(t)\| & \leq\|\phi(0)\|+\int_{0}^{t}\left\|f\left(x_{s}, \int_{-r_{0}}^{0} d \eta(s) g\left(x_{s}\left(-\tau\left(x_{s}\right)+s\right)\right)\right)\right\| d s \\
& \leq\|\phi\|+M \int_{0}^{t}\left\|x_{s}\right\| d s, \quad 0 \leq t \leq t^{*} .
\end{aligned}
$$

It follows that

$$
\hat{x}(t) \leq\|\phi\|+M \int_{0}^{t} \hat{x}(s) d s .
$$

Then the Gronwall inequality implies that

$$
\left\|x\left(t^{*}\right)\right\|=\hat{x}\left(t^{*}\right) \leq\|\phi\| e^{M t^{*}} \leq \delta^{*} e^{M t^{*}}<\delta_{0} e^{-M\left(r-t^{*}\right)} \leq \delta_{0} .
$$

This contradicts (3.2).

Lemma 3.3. Let

$$
G(\psi)=F(\psi)-L \psi .
$$

Then there exists a constant $N>0$ such that for each $\nu>0(\nu \leq 1)$, there is an $h(\nu)>0$ such that for $\psi \in B_{h(\nu)}$ with $\psi$ differentiable, we have

$$
\|G(\psi)\| \leq \nu N(\|\psi\|+\|\dot{\psi}\|)
$$


Proof. For any fixed $\psi \in C$, let $H(\theta)=\int_{-r_{0}}^{0} d \eta(s) g(\theta \psi(-\tau(\psi)+s))$ and $W(\theta)=$ $f(\theta \psi, H(\theta)), \theta \in[0,1]$. Then $W(1)=F(\psi)$ and $W(0)=0$. Therefore

$$
\begin{aligned}
F(\psi)= & W(1) \\
= & \int_{0}^{1} \dot{W}(\theta) d \theta \\
= & \int_{0}^{1} D_{\phi} f(\theta \psi, H(\theta)) \psi d \theta \\
& +\int_{0}^{1} D_{y} f(\theta \psi, H(\theta)) \int_{-r_{0}}^{0} d \eta(s) D g(\theta \psi(-\tau(\psi)+s)) \psi(-\tau(\psi)+s) d \theta .
\end{aligned}
$$

By (1.3) and (3.4) it is not difficult to verify that

$$
G(\psi)=\sum_{i=1}^{4} G_{i}(\psi)
$$

with

$$
\begin{aligned}
& G_{1}(\psi)= \int_{0}^{1}\left[D_{\phi} f(\theta \psi, H(\theta))-D_{\phi} f(0, \hat{\eta} g(0))\right] \psi d \theta, \\
& G_{2}(\psi)=\int_{0}^{1}\left\{\left[D_{y} f(\theta \psi, H(\theta))-D_{y} f(0, \hat{\eta} g(0))\right]\right. \\
&\left.\quad \cdot \int_{-r_{0}}^{0} d \eta(s) D g(\theta \psi(-\tau(\psi)+s)) \psi(-\tau(0)+s)\right\} d \theta, \\
& G_{3}(\psi)=\int_{0}^{1}\left\{D_{y} f(0, \hat{\eta} g(0))\right. \\
&\left.\cdot \int_{-r_{0}}^{0} d \eta(s)[D g(\theta \psi(-\tau(\psi)+s))-D g(0)] \psi(-\tau(0)+s)\right\} d \theta, \\
& G_{4}(\psi)=\int_{0}^{1}\left\{D_{y} f(\theta \phi, H(\theta)) \int_{-r_{0}}^{0} d \eta(s) D g(\theta \psi(-\tau(\psi)+s))\right. \\
&\cdot[\psi(-\tau(\psi)+s)-\psi(-\tau(0)+s)]\} d \theta .
\end{aligned}
$$

By continuous differentiability of $f$ and $g$ and continuity of $\tau$ for each $\nu>0(\nu \leq 1)$, we can choose an $h(\nu)>0$, such that for $\phi, \psi \in B_{h(\nu)}$,

$$
\begin{gathered}
\left\|D_{\phi} f\left(\phi, \int_{-r_{0}}^{0} d \eta(s) g(\phi(-\tau(\psi)+s))-D_{\phi} f(0, \hat{\eta} g(0))\right)\right\| \leq \nu, \\
\left\|D_{y} f\left(\phi, \int_{-r_{0}}^{0} d \eta(s) g(\phi(-\tau(\psi)+s))-D_{y} f(0, \hat{\eta} g(0))\right)\right\| \leq \nu, \\
\|D g(\phi)-D g(0)\| \leq \nu, \quad|\tau(\psi)-\tau(0)| \leq \nu .
\end{gathered}
$$


If follows from (3.5) that for $\psi \in B_{h(\nu)}$ with $\psi$ continuously differentiable,

$$
\begin{aligned}
\left\|G_{1}(\psi)\right\| & \leq \nu\|\psi\|, \\
\left\|G_{2}(\psi)\right\| & \leq \nu\|\eta\|(\|D g(0)\|+1)\|\psi\|, \\
\left\|G_{3}(\psi)\right\| & \leq \nu\|\eta\|\left\|D_{y} f(0, \hat{\eta} g(0))\right\|\|\psi\|, \\
\left\|G_{4}(\psi)\right\| & \leq \nu\left(\left\|D_{y} f(0, \hat{\eta} g(0))\right\|+1\right)(\|D g(0)\|+1)\|\eta\|\|\dot{\psi}\| .
\end{aligned}
$$

Now the lemma follows immediately from (3.6) if we set

$$
\begin{array}{r}
N=\max \left\{1+\|\eta\|\left(\|D g(0)\|+\left\|D_{y} f(0, \hat{\eta} g(0))\right\|+1\right),\right. \\
\|\eta\|(\|D f(0, \hat{\eta} g(0))\|+1)(\|D g(0)\|+1)\}
\end{array}
$$

Now we are in a position to prove Theorem 2.1.

Proof of Theorem 2.1. Let $T(t)$ be the semigroup generated by the solution of the linear system (2.2). Then for each given $\varepsilon \in(0, \alpha]$, there exists a constant $K_{1}(\varepsilon) \geq 1$ such that

$$
\|T(t) \phi\| \leq K_{1}(\varepsilon) e^{-\left(\alpha-\frac{\varepsilon}{2}\right) t}\|\phi\|, \quad \text { for } t \geq 0, \phi \in C .
$$

We choose $\nu \in(0,1]$ and $\delta_{0} \in(0, \delta]$ such that

$$
\nu \leq \frac{\varepsilon}{4 N(1+M) K_{1}(\varepsilon)}, \quad \delta_{0} \leq \min \left\{\frac{\delta}{K_{1}(\varepsilon)}, \frac{1}{M K_{1}(\varepsilon)}, \frac{h(\nu)}{K_{1}(\varepsilon)}\right\},
$$

where $\delta, M, N$ and $h(\nu)$ are defined as in Lemma 3.1 and Lemma 3.3. We now claim that, for $\phi \in B_{\delta^{*}}$ with $\delta^{*}<e^{-M r} \delta_{0}$,

$$
\left\|x_{t}(\phi)\right\|<K_{1}(\varepsilon) \delta_{0}, \quad t \in\left[0, t_{\phi}\right) .
$$

We prove this by contradiction. By Corollary 3.2 we have $t_{\phi}>r$ and $\left\|x_{t}(\phi)\right\|<\delta_{0}$, $t \in[0, r]$. So if inequality (3.7) is not true, then there must be a $t^{*}>r$ such that

$$
\left\|x_{t}(\phi)\right\|<K_{1}(\varepsilon) \delta_{0}, \quad t \in\left[0, t^{*}\right), \quad \text { and } \quad\left\|x_{t^{*}}(\phi)\right\|=K_{1}(\varepsilon) \delta_{0} .
$$

On the other hand, by the formula of variation of constants (see [8], Section 6.2, p. 173) for $t \in\left[r, t^{*}\right]$ we have

$$
\begin{aligned}
x_{t}(\phi) & =x_{t-r}\left(x_{r}(\phi)\right) \\
& =T(t-r) x_{r}(\phi)+\int_{0}^{t-r} T(t-r-s) X_{0} G\left(x_{s+r}(\phi)\right) d s .
\end{aligned}
$$

It follows from Lemma 3.1 and (3.8) that for $t \in\left[0, t^{*}\right]$,

$$
\|\dot{x}(t, \phi)\|=\left\|F\left(x_{t}(\phi)\right)\right\| \leq M\left\|x_{t}(\phi)\right\| \leq M K_{1}(\varepsilon) \delta_{0} .
$$

Therefore, $x_{t}(\phi)$ is differentiable for $t \in\left[r, t^{*}\right)$ and

$$
\left\|\dot{x}_{t}(\phi)\right\| \leq M K_{1}(\varepsilon) \delta_{0}, \quad t \in\left[r, t^{*}\right] .
$$


(3.9) and (3.10) imply that for $t \in\left[r, t^{*}\right]$,

$$
\begin{aligned}
&\left\|x_{t-r}\left(x_{r}(\phi)\right)\right\| \\
& \leq\|T(t-r)\|\left\|x_{r}(\phi)\right\|+\int_{0}^{t-r}\|T(t-r-s)\|\left\|G\left(x_{s+r}(\phi)\right)\right\| d s \\
& \leq K_{1}(\varepsilon) e^{-\left(\alpha-\frac{\varepsilon}{2}\right)(t-r)}\left\|x_{r}(\phi)\right\| \\
&+K_{1}(\varepsilon) \nu N \int_{0}^{t-r} e^{-\left(\alpha-\frac{\varepsilon}{2}\right)(t-r-s)}\left(\left\|x_{s+r}(\phi)\right\|+\left\|\dot{x}_{s+r}(\phi)\right\|\right) d s \\
& \leq K_{1}(\varepsilon) e^{-\left(\alpha-\frac{\varepsilon}{2}\right)(t-r)}\left\|x_{r}(\phi)\right\| \\
&+K_{1}(\varepsilon) \nu N \int_{0}^{t-r} e^{-\left(\alpha-\frac{\varepsilon}{2}\right)(t-r-s)}(1+M)\left\|x_{s}\left(x_{r}(\phi)\right)\right\| d s .
\end{aligned}
$$

By applying the Gronwall inequality we therefore have

$$
\begin{aligned}
\left\|x_{t^{*}}(\phi)\right\| & =\left\|x_{t^{*}-r}\left(x_{r}(\phi)\right)\right\| \\
& \leq K_{1}(\varepsilon) e^{-\left[\alpha-\frac{\varepsilon}{2}-\nu N(1+M) K_{1}(\varepsilon)\right]\left(t^{*}-r\right)}\left\|x_{r}(\phi)\right\| \\
& \leq K_{1}(\varepsilon) e^{-(\alpha-\varepsilon)\left(t^{*}-r\right)} \delta_{0} \\
& <K_{1}(\varepsilon) \delta_{0} .
\end{aligned}
$$

The last inequality contradicts (3.8). This proves our claim. As a consequence of (3.7) we have $t_{\phi}=\infty$ and that (3.7) is valid for all $t \geq r$. By applying again the Gronwall inequality to (3.11) and (3.3) we have

$$
\begin{aligned}
\left\|x_{t}(\phi)\right\| & =\left\|x_{t-r}\left(x_{r}(\phi)\right)\right\| \\
& \leq K_{1}(\varepsilon) e^{-(\alpha-\varepsilon)(t-r)}\left\|x_{r}(\phi)\right\| \\
& \leq K_{1}(\varepsilon) e^{-(\alpha-\varepsilon)(t-r)} e^{M r}\|\phi\| \\
& =K_{1}(\varepsilon) e^{(\alpha-\varepsilon+M) r} e^{-(\alpha-\varepsilon) t}\|\phi\|, \quad t \geq r,
\end{aligned}
$$

and

$$
\left\|x_{t}(\phi)\right\| \leq e^{M t}\|\phi\|=e^{(M+\alpha-\varepsilon) r} e^{-(\alpha-\varepsilon) t}\|\phi\|, \quad t \in[0, r] .
$$

If we let $K(\varepsilon)=K_{1}(\varepsilon) e^{(\alpha-\varepsilon+M) r}$, then (3.12) and (3.13) imply

$$
\left\|x_{t}(\phi)\right\| \leq K(\varepsilon) e^{-(\alpha-\varepsilon) t}\|\phi\|, \quad t \geq 0, \phi \in B_{\delta^{*}} .
$$

This proves part (i) of Theorem 2.1. For part (ii), we only give an outline of the proof. The idea of the proof is the same as for the state-independent delay FDE. In fact, if $\alpha>0$, then by using the same estimate as in the proof for part (i) and a similar argument to the usual FDE (for instance, see Theorem 1.1, Chapter 10 in $[8]$ ), we are able to show that equation (2.1) has a nonzero solution $x(t)$ which is defined for all $t \leq 0$ and

$$
\lim _{t \rightarrow-\infty} x(t)=0 .
$$

Thus the zero solution is unstable. 


\section{EXAMPLES}

Example 1. Consider the equation

$$
\dot{x}(t)=h\left(\int_{-r_{0}}^{0} d \eta_{1}(s) x(t+s), \int_{-r_{0}}^{0} d \eta_{2}(s) x\left(t-\tau\left(x_{t}\right)+s\right)\right),
$$

where $x(t) \in \mathbb{R}^{n}, h: \mathbb{R}^{n} \times \mathbb{R}^{n} \rightarrow \mathbb{R}^{n}$ is differentiable, $\tau: C \rightarrow \mathbb{R}^{+}$is continuous and $\eta_{1}, \eta_{2}$ are of bounded variation on $[-r, 0]$. Suppose that there is an $x^{*} \in \mathbb{R}^{n}$ such that

$$
h\left(\int_{-r_{0}}^{0} d \eta_{1}(s) x^{*}, \int_{-r_{0}}^{0} d \eta_{2}(s) x^{*}\right)=0 .
$$

Then $x^{*}$ is an equilibrium of (4.1). By Theorem 2.1 the formal linearization at $x^{*}$ is

$$
\dot{x}(t)=L x_{t},
$$

with

$$
\begin{aligned}
L \phi= & D_{1} h\left(\int_{-r_{0}}^{0} d \eta_{1}(s) x^{*}, \int_{-r_{0}}^{0} d \eta_{2}(s) x^{*}\right) \int_{-r_{0}}^{0} d \eta_{1}(s) \phi(s) \\
& +D_{2} h\left(\int_{-r_{0}}^{0} d \eta_{1}(s) x^{*}, \int_{-r_{0}}^{0} d \eta_{2}(s) x^{*}\right) \int_{-r_{0}}^{0} d \eta_{2}(s) \phi\left(-\tau\left(x^{*}\right)+s\right) .
\end{aligned}
$$

Here $D_{i} h, i=1,2$, is the derivative of $h$ with respect to the $i$ th variable, respectively. A simple equation of the form (4.1) is

$$
\dot{x}(t)=A x(t)+B x\left(t-\tau\left(x_{t}\right)\right),
$$

in which $A$ and $B$ are $n \times n$ matrices. In this case $h$ takes the form

$$
h(x, y)=A x+B y,
$$

and

$$
\int_{-r_{0}}^{0} d \eta_{1}(s) \phi(s)=\int_{-r_{0}}^{0} d \eta_{2}(s) \phi(s)=\phi(0)
$$

It is clear that $x^{*}=0$ is an equilibrium and

$$
\begin{aligned}
L \phi & =D_{1} h(0,0) \int_{-r_{0}}^{0} d \eta_{1}(s) \phi(s)+D_{2} h(0,0) \int_{-r_{0}}^{0} d \eta_{2}(s) \phi\left(-\tau\left(x^{*}\right)+s\right) \\
& =A \phi(0)+B \phi(-\tau(0)) .
\end{aligned}
$$

Hence the formal linearization at zero is

$$
\dot{x}(t)=A x(t)+B x(t-\tau(0)) .
$$

Example 2. Consider the functional equation

$$
x(t)=\int_{t-\xi(x(t))}^{t} g(x(s)) d s,
$$

where $g: \mathbb{R} \rightarrow \mathbb{R}$ and $\xi: \mathbb{R} \rightarrow \mathbb{R}^{+}$are continuously differentiable. (4.2) has been studied in [3]. By differentiating (4.2) we can obtain a differential equation

$$
\dot{x}(t)=\frac{g(x(t))-g(x(t-\xi(x(t))))}{1-\xi^{\prime}(x(t)) g(x(t-\xi(x(t))))} .
$$


Note that equation (4.3) is not equivalent to (4.2). It is clear that every solution of (4.2) is a solution of (4.3) but the reverse is not true. In fact any constant function is a solution of (4.3) but clearly it may not necessarily be a solution of (4.2). Nevertheless we can use (4.3) to study the stability for equation (4.2). Suppose that $\bar{x}$ is an equilibrium of (4.2), that is, $\bar{x}=g(\bar{x}) \xi(\bar{x})$. We formally linearize (4.3) around $\bar{x}$. Now (4.3) is a special case of (4.1) with

and

$$
h(x, y)=\frac{g(x)-g(y)}{1-\xi^{\prime}(x) g(y)},
$$

$$
\int_{-r_{0}}^{0} d \eta_{1}(s) \phi(s)=\int_{-r_{0}}^{0} d \eta_{2}(s) \phi(s)=\phi(0)
$$

Therefore we have the corresponding linear system at $\bar{x}$ :

$$
\begin{aligned}
\dot{x}(t) & =h_{x}(\bar{x}, \bar{x}) x(t)+h_{y}(\bar{x}, \bar{x}) x(t-\xi(\bar{x})) \\
& =\frac{g^{\prime}(\bar{x})}{1-g(\bar{x}) \xi^{\prime}(\bar{x})}[x(t)-x(t-\xi(\bar{x}))] .
\end{aligned}
$$

As we mentioned above, the stability of the zero solution of (4.4) is not equivalent to the stability of $\bar{x}$ as an equilibrium of (4.2) ((4.4) always has a zero eigenvalue!). However, we have ${ }^{2}$

The equilibrium $\bar{x}$ is asymptotically stable if zero is a simple eigenvalue, and all other eigenvalues of (4.4) have negative real part.

\section{REFERENCES}

[1] W. G. Aiello, H. I. Freedman, and J. Wu, Analysis of a model representing stage-structured population growth with state-dependent time delay, SIAM J. Appl. Math. 52, 1992, 855-869. MR 93j:92025

[2] W. Alt, Periodic solutions of some autonomous differential equations with variable time delay, Lecture Notes in Mathematics, Vol. 730, Springer-Verlag, 1979. MR 80i:34118

[3] J. Belair, Population models with state-dependent delays, In Mathematical Population Dynamics (O. Arino, D. E. Axelrod, and M. Kimmel, Eds.), Marcel Dekker, Inc., New YorkBasel-Hong Kong, 1991, 165-176. MR 94e:92022

[4] S. P. Blythe, R. M. Nisbet, and W. S. C. Gurney, The dynamics of population models with distributed maturation periods, Theor. Pop. Biology 25, 1984, 289-311. MR 85h:92030

[5] K. L. Cooke, Functional differential equations: some models and perturbation problems, In Differential Equations and Dynamical Systems (J. K. Hale and J. P. LaSalle, Eds.), Academic Press, New York, 1967. MR 36:5461

[6] K. L. Cooke and W. Huang, A theorem of George Seifert and an equation with state-dependent delay, In Delay and Differential Equations (A. M. Fink, R. K. Miller, and W. Kliemann, Eds.), World Scientific, Singapore, 1992, 65-77. MR 93d:34128

[7] J. A. Gatica and P. Waltman, Existence and uniqueness of solutions of a functional differential equation modeling thresholds, Nonlinear Analysis T.M.A. 8, 1984, 1215-1222. MR 86h:34080

[8] J. K. Hale, Theory of Functional Differential Equations, Springer-Verlag, New York, 1977. MR 58:22904

[9] F. C. Hoppensteadt and P. Waltman, A flow mediated control model of respiration, In Some Mathematical Questions in Biology, Vol. 12, Lectures on Mathematics in the Life Sciences, 1979, 211-218. MR 82k:92026

[10] Y. Kuang and H. L. Smith, Slowly oscillating periodic solutions of autonomous statedependent delay equations, IMA Preprint Series \#754, Minneapolis, 1990.

\footnotetext{
${ }^{2}$ The detailed proofs of part (ii) of Theorem 2.1 and our last result will be given in a later paper.
} 
[11] J. A. J. Metz and O. Diekmann, The Dynamics of Physiologically Structured Populations, Lecture Notes in Biomathematics 68, Springer-Verlag, 1986. MR 88b:92049

[12] H. L. Smith, Threshold delay differential equations are equivalent to FDE's, preprint.

[13] H. L. Smith, Reduction of structured population models to threshold-type delay equations and functional differential equations: a case study, Math. Biosci. 113 (1993), 1-23. MR 93k:20054

Department of Mathematics, Pomona College, Claremont, California 91711

E-mail address: kcooke@pomona.edu

Department of Mathematics, University of Alabama in Huntsville, Huntsville, AlABAMA 35899

E-mail address: huang@math.uah.edu 